\title{
Rhabdomyolysis complicating unrecognized hypophosphatemia in an alcoholic patient
}

\author{
Deepali Kumar MD ${ }^{1}$, Finlay A McAlister MD FRCPC ${ }^{2}$
}

\begin{abstract}
D Kumar, FA McAlister. Rhabdomyolysis complicating unrecognized hypophosphatemia in an alcoholic patient. Can J Gastroenterol 1999;13(2):165-167. Rhabdomyolysis occurring as a complication of hypophosphatemia has been infrequently described. A 58-year-old male with a history of daily alcohol consumption presented with two generalized tonic clonic seizures secondary to hypovolemic hyponatremia. He was volume-resuscitated, and antiepileptic medication was administered. After three days of hospitalization, the patient developed severe rhabdomyolysis despite the absence of further seizure activity. Serum phosphate levels were depressed. He was treated with intravenous mannitol, alkaline diuresis, and intravenous and oral phosphate supplementation. He recovered uneventfully. Hypophosphatemia can potentially lead to multisystem organ dysfunction including severe rhabdomyolysis. It is, therefore, important to maintain a low threshold for measuring serum phosphate levels in patients admitted to hospital.
\end{abstract}

Key Words: Hypophosphatemia, Rhabdomyolysis

\section{Rhabdomyolyse compliquant l'hypophosphatémie non reconnue chez un patient alcoolique}

RÉSUMÉ : La rhabdomyolyse compliquant l'hypophosphatémie est un phénomène rarement décrit. Un homme de 58 ans consommant de l'alcool quotidiennement s'est présenté pour deux accès de convulsions tonico-cloniques généralisées, suite à une hyponatrémie hypovolémique. On a réagi en lui administrant du volume et des antiépileptiques. Après trois jours d'hospitalisation, le patient a présenté une grave rhabdomyolyse, malgré l'absence d'autres crises de convulsions. Ses taux de phosphate sériques étaient bas. Il a été traité au moyen de mannitol intraveineux, diurèse alcaline et suppléments intraveineux et oraux de phosphate. Il a récupéré sans complications. L'hypophosphatémie peut entraîner une dysfonction pluriorganique, y compris une rhabdomyolyse grave. Il est donc important de maintenir un seuil bas pour la mesure des taux de phosphate sériques chez les patients hospitalisés.
$\mathrm{P}$ hosphorus is an essential component of cellular structure and function, and is the major intracellular anion. Total body deficits of phosphorus can result from poor intake or increased loss, and has been reported to be present in $10 \%$ to $30 \%$ of hospitalized patients $(1,2)$. Although hypophosphatemia may lead to potentially life-threatening sequelae, which are initially subclinical, the measurement and replacement of phosphate are often overlooked.

We describe a case of severe rhabdomyolysis developing in a chronic alcohol user as a result of unrecognized hypophosphatemia.

\section{CASE PRESENTATION}

A 58-year-old male presented after two witnessed generalized tonic clonic seizures. He had a past history of hyper- tension, chronic obstructive pulmonary disease and depression, and had been consuming approximately $7 \mathrm{U}$ of alcohol daily for over 35 years. There was no personal or family history of metabolic myopathy or rhabdomyolysis, His medications included theophylline, inhaled bronchodilators, prednisone, a thiazide diuretic and potassiumsparing diuretic combination product, and an angiotensinconverting enzyme inhibitor. He had stopped drinking three days before admission due to weakness and a fall.

Physical examination on presentation revealed tachycardia (110 beats/min), hypotension (systolic blood pressure $60 \mathrm{mmHg}$ ) and tachypnea ( 28 breaths/min). He was afebrile, postictal and grossly dehydrated; however, the results of the remainder of the examination were essentially unremarkable. Specifically, there were no focal neurological signs.

\footnotetext{
${ }^{1}$ The Division of General Internal Medicine, Ottawa Civic Hospital, Ottawa, Ontario; ${ }^{2}$ Centre for Evidence-Based Medicine, Nuffield Department of Medicine, Level 5, John Radcliffe Hospital, Headington, Oxford, United Kingdom

Correspondence: Dr F McAlister, Centre for Evidence-Based Medicine, Nuffield Department of Medicine, Level 5, John Radcliffe Hospital, Headington, Oxford, United Kingdom OX3 9DU. Telephone 44-1865-221321, fax 44-1865-222901, e-mail fmcalister@lri.ca
} 


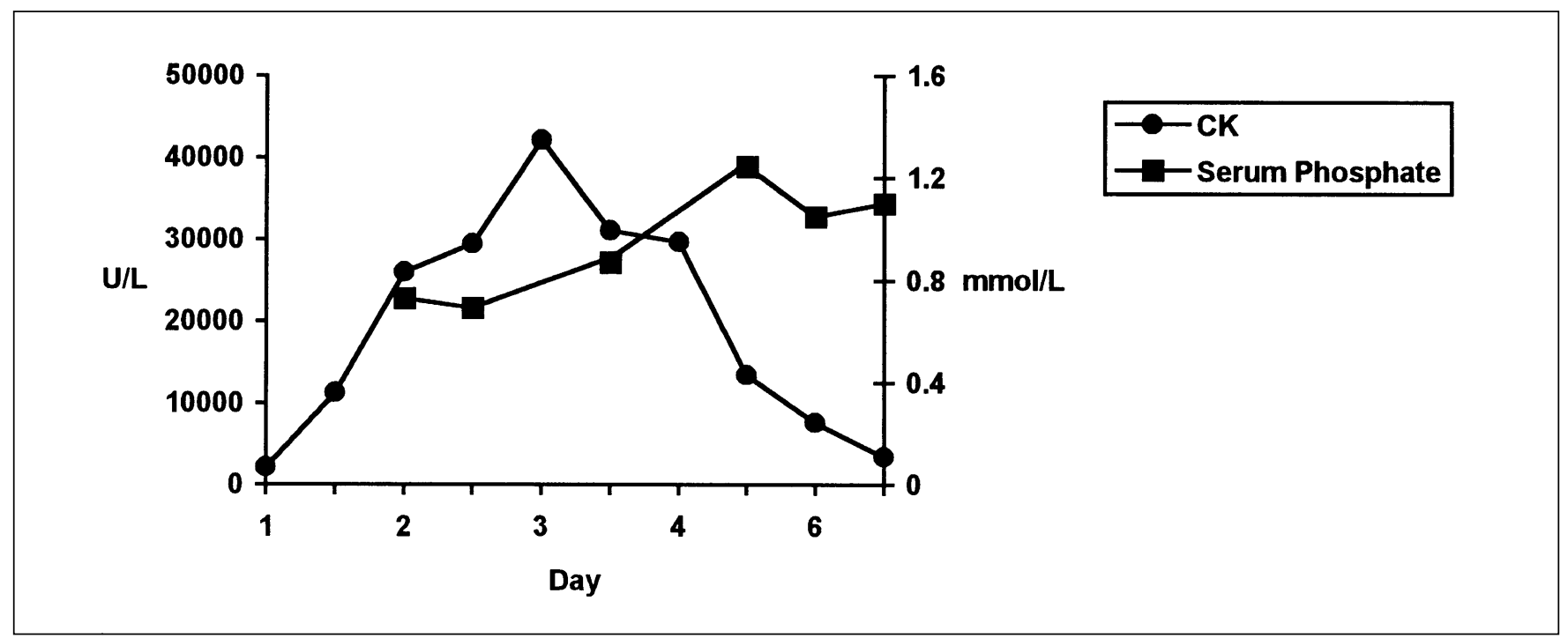

Figure 1) Relationship between serum creatinine kinase (CK) and serum phosphate

Laboratory investigations on admission revealed hyponatremia $(108 \mathrm{mmol} / \mathrm{L})$, lactic acidosis (serum lactate $14.8 \mathrm{mmol} / \mathrm{L}$, serum bicarbonate $5 \mathrm{mmol} / \mathrm{L}$, anion gap $25 \mathrm{mmol} / \mathrm{L}$ and osmolal gap 7), renal failure (creatinine $300 \mu \mathrm{mol} / \mathrm{L}$ with urea $21 \mathrm{mmol} / \mathrm{L}$ ) and leucocytosis (white cell count of 31,000 with a left shift). His creatine kinase (CK) level was elevated at $2156 \mathrm{U} / \mathrm{L}$ (normal range 20 to $215 \mathrm{U} / \mathrm{L}$ in the authors' laboratory) $8 \mathrm{~h}$ postseizure. Serum calcium, magnesium, potassium and glucose, results of liver function tests, hemoglobin and platelet levels, and results of urinalysis were within normal limits. An electrocardiogram demonstrated sinus tachycardia with no ischemic changes. Computed tomography of the brain revealed diffuse atrophy with no focal lesions; lumbar puncture confirmed normal cerebrospinal fluid. Chest radiograph was unremarkable.

The patient's seizures were felt to be a result of his hypovolemic hyponatremia, and he was volume-resuscitated with normal saline. Intravenous phenytoin, corticosteroids and antibiotics were also administered. The antibiotics were discontinued in $48 \mathrm{~h}$, when all cultures were negative.

The patient's vital signs and metabolic abnormalities rapidly normalized (serum creatinine declined to 91 $\mu \mathrm{mol} / \mathrm{L}$ within $48 \mathrm{~h}$ ), he had no further seizures and he regained consciousness. He was started back on a normal diet by the second day of admission and, aside from general weakness, was feeling well. However, it was noted that his CK levels continued to rise after admission despite the absence of further seizure activity. By the second day of hospitalization, his CK level was $11,242 \mathrm{U} / \mathrm{L}$, by day three it was $42,114 \mathrm{U} / \mathrm{L}$ and by day five it was $13,395 \mathrm{U} / \mathrm{L}$.

A search for what caused the elevated (and rising) CK levels was unrewarding; he was not receiving any intramuscular injections, his thyroid-stimulating hormone level was normal, and he had no apparent muscle trauma, tenderness, tightness or swelling. It was felt that he had subclinical rhabdomyolysis, and his metabolites were rechecked. His serum sodium was $128 \mathrm{mmol} / \mathrm{L} 72 \mathrm{~h}$ after admission, and the only other abnormality was hypophosphatemia $(0.71 \mathrm{mmol} / \mathrm{L}$; normal range in the authors' laboratory 0.80 to $1.60 \mathrm{mmol} / \mathrm{L}$ ). His $\mathrm{CK}$ and serum phosphate levels are illustrated in Figure 1. Because his urine was positive for myoglobin, he was treated with alkaline diuresis and mannitol. In addition, he was given intravenous $(37.8 \mathrm{mmol} / \mathrm{day})$ and oral $(96.8 \mathrm{mmol} / \mathrm{day})$ phosphate supplementation. The patient recovered completely within seven days.

\section{DISCUSSION}

Our patient developed rhabdomyolysis more than $48 \mathrm{~h}$ after presenting with two brief seizures. In the absence of another explanation, we assume that his rhabdomyolysis was secondary to hypophosphatemia. It has been well documented that serum phosphate levels are poorly reflective of intracellular phosphate levels (3), and the occurrence of rhabdomyolysis is not closely correlated with the serum phosphate concentration (2). Moreover, because phosphate is released from cells when rhabdomyolysis occurs, the serum phosphate level is artificially elevated when the serum CK level is increased. Although Singhal et al (2) found that asymptomatic rhabdomyolysis was a common complication of the hypophosphatemic state, they documented the difficulty in discerning which hypophosphatemic patients would develop the complication (and the difficulty in diagnosing it because none of the patients had classical symptoms of rhabdomyolysis). While they were able to identify a clinical prediction rule using six biochemical variables identified by logistic regression techniques, their work has not been replicated in other cohorts and, thus, remains hypothesis-generating rather than definitive (4). At this time, it seems most appropriate to exercise a high index of suspicion for rhabdomyolysis in all 
hypophosphatemic patients rather than to rely on an unproven prediction rule.

Hypophosphatemia reflects depleted total body stores of phosphate (due to poor intake or excessive loss of phosphate) or cellular shifts. Nutritional deficiency of phosphate is generally uncommon due to the ubiquitous nature of phosphate in natural foods. Compensatory mechanisms act to maintain phosphate balance in cases of dietary deficiency, and include decreased renal excretion, reduced intestinal secretion and increased bone reabsorption of phosphate. However, phosphate losses can occur via renal and nonrenal pathways. Sources of nonrenal losses include prolonged nasogastric suction, vomiting or chronic diarrhea. Renal losses can result from primary renal tubular defects such as proximal renal tubular acidosis or secondary tubular dysfunction as is seen with hyperparathyroidism. Clinically significant hypophosphatemia is usually due to transcellular shifts. In hospitalized patients, these shifts often result from 'refeeding syndrome'. This syndrome, as described by Knochel et al (5) and Knochel (6), results from administration of carbohydrates and amino acids to malnourished patients and the subsequent increase in cellular uptake of phosphate. If insufficient phosphate is given with the feeding, profound hypophosphatemia can result, usually around 48 to $72 \mathrm{~h}$ after the start of refeeding. Other causes of phosphate redistribution include metabolic or respiratory alkalosis, excess catecholamines and increased glucagon levels. The refeeding syndrome is particularly likely to occur in malnourished alcoholic patients, and given the time frame, we postulate that our patient's hypophosphatemia could have been triggered through this pathway.

Alcoholics are particularly prone to hypophosphatemia for a variety of reasons. First, poor nutrition, or nausea and vomiting may lead to a dietary phosphate deficiency. Second, pancreatic insufficiency with malabsorption and steatorrhea may result in secondary hyperparathyroidism and renal phosphate loss (because parathyroid hormone exerts a phosphaturic action on the renal tubule). Phosphaturia may also be enhanced by ketonuria, hypokalemia and hypomagnesemia, all of which are common in malnourished alcoholic patients. Transcellular shifts can also holic patients are found to have hypophosphatemia on laboratory testing, most have a total body phosphate deficit $(7,8)$. Moreover, alcoholic patients appear to be at particularly high risk of rhabdomyolysis if they develop hypophosphatemia, likely because of pre-existing subclinical myopathy from the direct toxic effects of ethanol. As pointed out by Knochel (9), in the setting of pre-existing cellular injury, hypophosphatemia results in cellular death because of the reduced capability of the cell to regenerate ATP.

In addition to rhabdomyolysis, other possible consequences of hypophosphatemia include myocardial depression; respiratory muscle weakness; metabolic encephalopathy (manifesting as confusion or seizures); peripheral neuropathy; osteomalacia and vitamin D-resistant rickets; hemolysis, and platelet or leukocyte dysfunction; and a chronic proximal myopathy distinct from acute rhabdomyolysis.

Serum phosphate measurement is often overlooked in patients admitted to hospital, and the present case emphasizes the often asymptomatic nature of even the lifethreatening sequelae of hypophosphatemia. We caution the reader to maintain a high index of suspicion for deficiency of this often forgotten anion.

ACKNOWLEDGEMENTS: Dr McAlister is supported by the Alberta Heritage Foundation for Medical Research and the Medical Research Council of Canada.

\section{REFERENCES}

1. Betro MG, Pain RW. Hypophosphataemia and hyperphosphataemia in a hospital population. Br Med J 1972;1:273-6.

2. Singhal PC, Kumar A, Desroches L, Gibbons N, Mattana J. Prevalence and predictors of rhabdomyolysis in patients with hypophosphatemia. Am J Med 1992;92:458-64.

3. Crook M. Phosphate: an abnormal anion. Br J Hosp Med 1994;52:200-3.

4. Laupacis A, Sekar N, Stiell IG. Clinical prediction rules. A review and suggested modifications of methodological standards. JAMA 1997;277:488-94.

5. Knochel JP, Barcenas C, Cotton JR, Fuller TJ, Haller R, Carter NW. Hypophosphatemia and rhabdomyolysis. J Clin Invest 1978;62:1240-6.

6. Knochel JP. The pathophysiology and clinical characteristics of severe hypophosphatemia. Arch Intern Med 1977;137:203-20. result from the excessive catecholamine release seen in delirium tremens, or the respiratory alkalosis commonly associated with liver disease, alcohol withdrawal or sepsis Thus, although only about one-third of hospitalized alco-
7. Elisaf M, Markos M, Tsianos EV, Siamopoulos KC. Acid-base and electrolyte abnormalities in alcoholic patients. Miner Electrolyte Metab 1994;20:274-81.

8. Ryback RS, Eckardt MJ, Pautler CP. Clinical relationships between serum phosphorus and other blood chemistry values in alcoholics. Arch Intern Med 1980;140:673-7

9. Knochel JP. Hypophosphatemia and rhabdomyolysis. Am J Med 1992;92:455-7. 


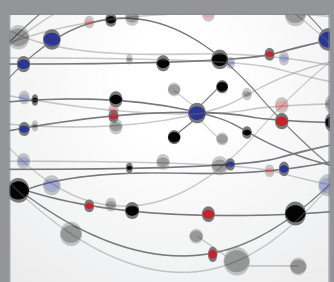

The Scientific World Journal
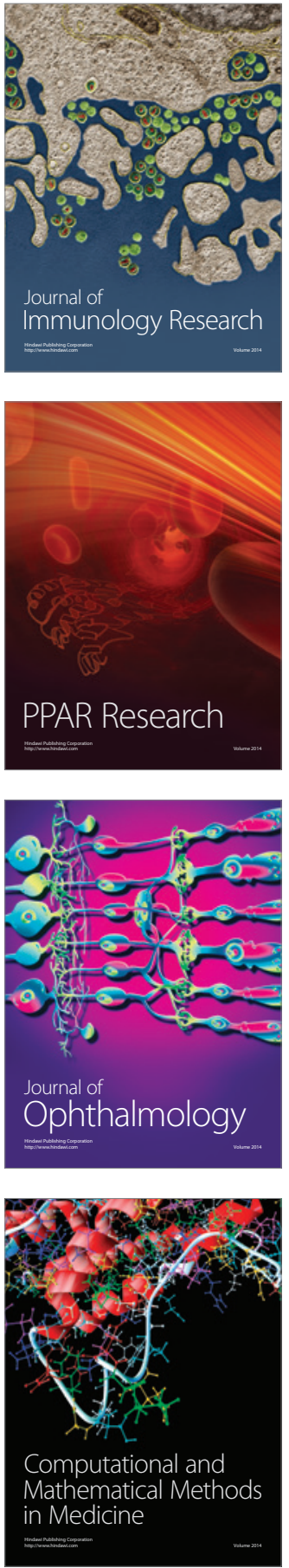

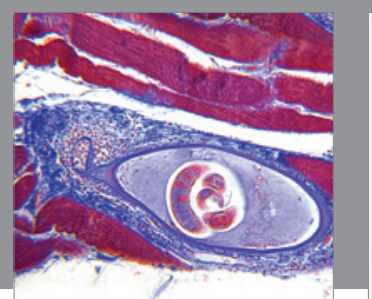

Gastroenterology Research and Practice

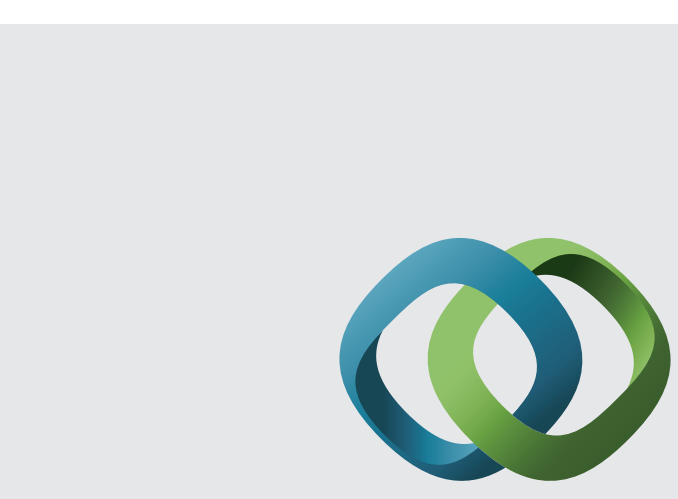

\section{Hindawi}

Submit your manuscripts at

http://www.hindawi.com
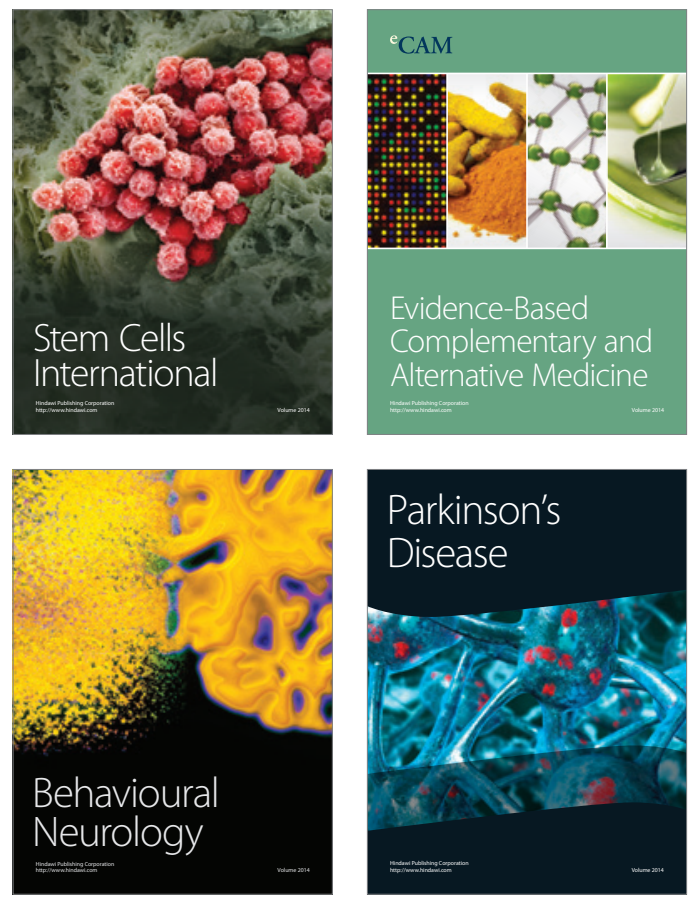
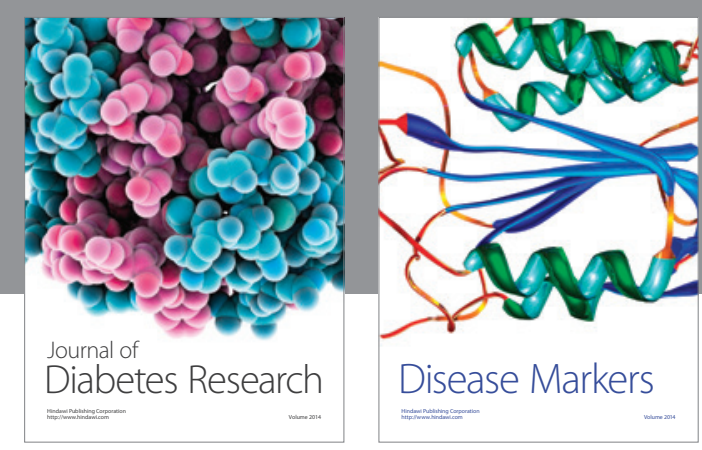

Disease Markers
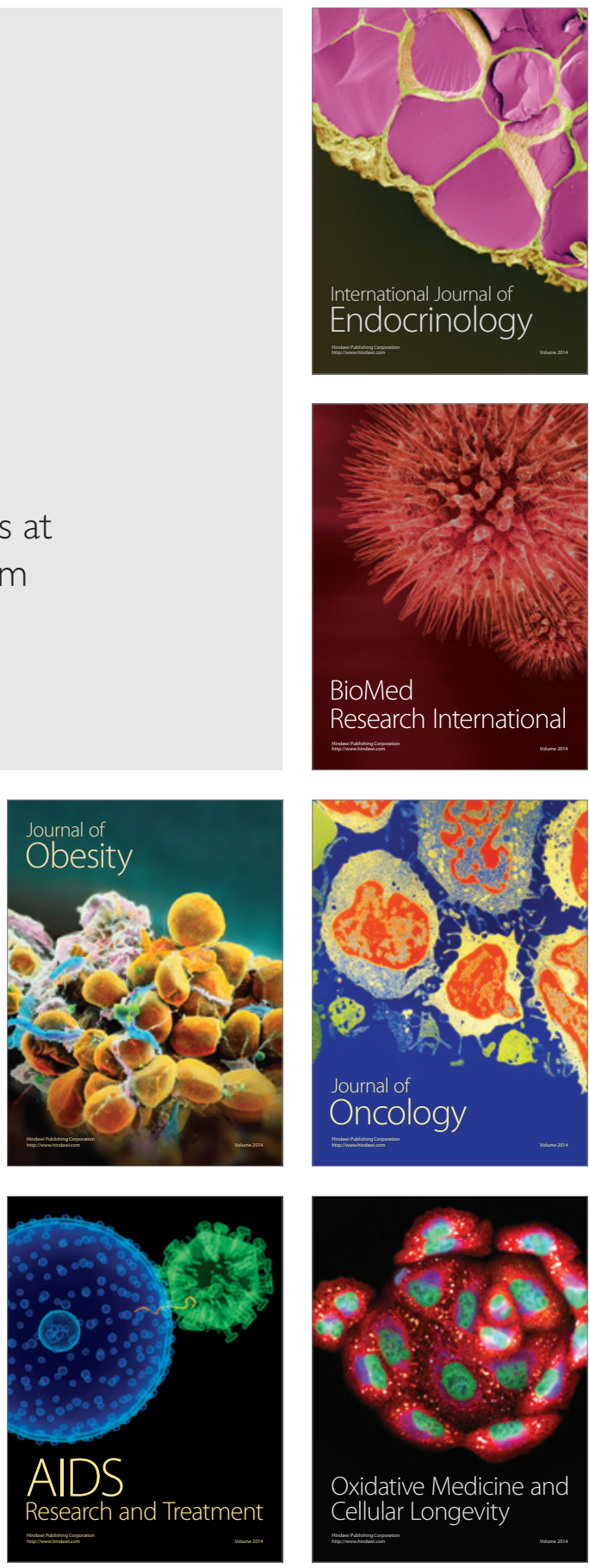